\title{
Comprehensive Axillary Evaluation in Neoadjuvant Chemotherapy Patients With Ultrasonography and Sentinel Lymph Node Biopsy
}

\author{
Amina Khan, MD, ${ }^{1}$ Michael S. Sabel, MD,${ }^{1}$ Alexis Nees, MD, ${ }^{2}$ Kathleen M. Diehl, MD, ${ }^{1}$ \\ Vincent M. Cimmino, MD, ${ }^{1}$ Celina G. Kleer, MD, ${ }^{3}$ Anne F. Schott, MD, ${ }^{4}$ \\ Dan F. Hayes, MD, ${ }^{4}$ Alfred E. Chang, MD, ${ }^{1}$ and Lisa A. Newman, MD, MPH ${ }^{1}$ \\ ${ }^{1}$ Department of Surgery, Comprehensive Cancer Center, University of Michigan, 1500 East Medical Center Drive, Ann Arbor, \\ Michigan 48109 \\ ${ }^{2}$ Department of Radiology, Comprehensive Cancer Center, University of Michigan, Ann Arbor, Michigan 48109 \\ ${ }^{3}$ Department of Pathology, Comprehensive Cancer Center, University of Michigan, Ann Arbor, Michigan 48109 \\ ${ }^{4}$ Department of Medical Oncology, Comprehensive Cancer Center, University of Michigan, Ann Arbor, Michigan 48109
}

\footnotetext{
Received September 10, 2004; accepted April 4, 2005; published online August 4, 2005.

Address correspondence and reprint requests to: Lisa A. Newman, MD, MPH; E-mail: lanewman@umich.edu

Published by Springer Science+Business Media, Inc. (c) 2005 The Society of Surgical Oncology, Inc.
}

\begin{abstract}
Background: There is ongoing debate regarding the optimal sequence of sentinel lymph node (SLN) biopsy and neoadjuvant chemotherapy (CTX) for breast cancer. We report the accuracy of comprehensive pre-neoadjuvant CTX and post-neoadjuvant CTX axillary staging via ultrasound imaging, fine-needle aspiration (FNA) biopsy, and SLN biopsy.

Methods: From 2001 to 2004, 91 neoadjuvant CTX patients at the University of Michigan Comprehensive Cancer Center underwent axillary staging by ultrasonography, ultrasoundguided FNA biopsy, SLN biopsy, or a combination of these.

Results: Axillary staging was pathologically negative by pre-neoadjuvant CTX SLN biopsy in 53 cases (58\%); these patients had no further axillary surgery. In 38 cases (42\%), axillary metastases were confirmed at presentation by either ultrasound-guided FNA or SLN biopsy. These 38 patients underwent completion axillary lymph node dissection (ALND) after delivery of neoadjuvant CTX. Follow-up lymphatic mapping was attempted in 33 of these cases, and the SLN was identified in 32 (identification rate, 97\%). One third of these cases were completely node negative on ALND. Residual metastatic disease was identified in 22 cases, and the SLN was falsely negative in $1(4.5 \%)$.

Conclusions: Patients receiving neoadjuvant CTX can have accurate axillary nodal staging by ultrasound-guided FNA or SLN biopsy. In cases of documented axillary metastasis at presentation, repeat axillary staging with SLN biopsy can document the post-neoadjuvant CTX nodal status. This strategy optimizes pre-neoadjuvant CTX and post-neoadjuvant CTX staging information by distinguishing the patients who are node negative at presentation from those who have been downstaged to node negativity and offers the potential for avoiding unnecessary ALNDs in both of these patient subsets.
\end{abstract}

Key Words: Neoadjuvant chemotherapy—Breast cancer-Axilla—Ultrasound-Sentinel node. the surgical management of breast cancer. Most notable has been the introduction in the mid 1990s of sentinel lymph node (SLN) biopsy, ${ }^{1-3}$ which has replaced conventional axillary lymph node dissection (ALND) as the initial procedure of choice in many institutions for the accurate staging of breast cancer. 
Despite varying lymphatic mapping techniques used worldwide, identification rates remain consistently higher than $91 \%$, with false negatives estimated at $8 \%$; with more extensive experience, these rates approach $100 \%^{5-7}$ and $<5 \%,^{7,8}$ respectively. A pathologically negative SLN is now widely accepted as reliably identifying the node-negative breast cancer patient who does not need to face the morbidity of a standard ALND. It should be noted, however, that data continue to mature from long-term prospective trials regarding outcomes in patients treated by SLN biopsy alone. ${ }^{9}$

The past 10 years have also witnessed the expansion of neoadjuvant chemotherapy (CTX) programs. Although induction CTX is the standard of care for patients with locally advanced breast cancer, the advantages of primary breast tumor downstaging, with improved eligibility for breast-conservation therapy and the ability to more rapidly assess chemosensitivity, have motivated the development of protocols that offer primary systemic therapy to patients with earlier-stage, operable breast cancer as well. An area of persistent controversy has been how to optimally integrate SLN biopsy into the neoadjuvant CTX treatment sequence: should the staging SLN biopsy be performed before or after the delivery of induction CTX? Potential concerns with either approach involve lymphatic mapping accuracy in patients with relatively larger T2 and T3 tumors, the effect of CTX on intramammary lymphatics, and effect of CTX on axillary nodal metastases.

The earliest reported studies involved performing the SLN biopsy after the delivery of neoadjuvant CTX, concomitantly with the definitive local breast therapy and in conjunction with a standard ALND. These studies have demonstrated mixed results, with false-negative rates ranging from $0 \%$ to $33 \%$, and the effects of a significant learning curve can be seen. ${ }^{10-15}$ Many clinicians remain dissatisfied with the available data on the accuracy of post-neoadjuvant CTX SLN biopsy and have opted instead for a staging SLN biopsy performed before delivery of the neoadjuvant CTX. The primary disadvantages to this approach, however, are the requirement for an additional surgical procedure at the time of presentation and the fact that the patients identified as being node positive by this pre-CTX SLN biopsy are then committed to undergoing the completion ALND, thereby negating some of the benefits of CTX downstaging.

Our institution sought to address this issue with a comprehensive approach to the axilla that uses ultrasound nodal imaging and lymphatic mapping in the pre-neoadjuvant CTX and post-neoadjuvant
CTX setting. With this strategy, we specifically evaluated the accuracy of lymphatic mapping performed after the delivery of neoadjuvant CTX in patients who had pathologically proven axillary metastases at presentation on the basis of either an ultrasoundguided fine-needle aspiration (FNA) or a SLN biopsy performed at the time of diagnosis.

\section{METHODS}

From March 2001 to May 2004, 91 patients with stage I, II, or IIIA breast cancers registered in a prospectively maintained breast cancer database underwent pre-neoadjuvant CTX axillary staging at the University of Michigan Comprehensive Cancer Center. Percutaneous core needle biopsy was the primary method of establishing the pathologic diagnosis of breast cancer. Diagnostic and therapeutic management strategies for all patients were reviewed at the Cancer Center multidisciplinary tumor board conference. The conduct of this study, with retrospective review of all surgical and pathologic records of appropriate patients, was approved by the University of Michigan Institutional Review Board.

Axillary staging by SLN biopsy performed before the delivery of neoadjuvant CTX was adopted as a routine practice by the Breast Oncology Program at the University of Michigan until approximately December 2001. From that point forward, the initial axillary evaluation included ultrasound imaging of the nodal basin, with ultrasound-guided FNA biopsy of any abnormal-appearing lymph nodes. If the ultrasound imaging was negative for evidence of metastases, the patient then proceeded to undergo definitive pre-neoadjuvant CTX axillary staging by SLN biopsy. If the patient was found to have axillary metastases on the basis of either the ultrasound-guided or SLN biopsy, then she proceeded to receive her neoadjuvant CTX, but a completion ALND was performed after the neoadjuvant CTX was delivered, concomitantly with the definitive breast surgery (lumpectomy or mastectomy). Lymphatic mapping and SLN biopsy was performed in conjunction with this completion ALND. If the definitive pre-neoadjuvant CTX SLN biopsy had negative results, then the patient proceeded to receive her neoadjuvant CTX, and no further axillary surgery was performed. The neoadjuvant CTX regimen included doxorubicin in all cases.

Axillary ultrasonography was performed by dedicated breast-imaging staff, and all FNA biopsy results were interpreted by an experienced cytopathologist. Lymph nodes were deemed appropriate for ultra- 
sound-guided FNA biopsy if they had irregular morphology (as indicated by cortical thickening), compressed fatty hilum, or complete loss of the fatty hilum.

Lymphatic mapping was performed as a dual-agent procedure (subareolar ${ }^{99 \mathrm{~m}} \mathrm{Tc}-$ labeled sulfur colloid 1 day before surgery and isosulfan blue dye injected during surgery) as the preferred method unless the patient specifically declined isotope injection. Five patients refused the isotope injection in conjunction with the mapping procedure performed concomitantly with the ALND after delivery of the neoadjuvant CTX. The SLNs were evaluated histopathologically by serial sectioning and hematoxylin and eosin staining; no immunohistochemistry analyses for cytokeratin were performed. Non-SLNs were evaluated by standard bivalving and staining of representative sections.

\section{RESULTS}

\section{Overall Study Population}

Table 1 shows the clinicopathologic features of the 91 neoadjuvant CTX cases evaluated in this study at the time of diagnosis.

\section{Node-Negative Patients}

Initial pre-neoadjuvant CTX axillary evaluation consisting of ultrasound imaging and SLN biopsy was completely negative for evidence of metastatic disease in 53 cases (58\%). These patients underwent no additional axillary surgery, and at a median follow-up of 14 months (range, 1-39 months), there have been no locoregional relapses in this subset of patients with node-negative disease at presentation.

\section{Node-Positive Patients}

The final study population consisted of 38 neoadjuvant CTX patients who were identified as having node-positive disease at presentation; 18 of the metastatic nodes were identified by SLN biopsy and 20 by ultrasound-guided FNA biopsy. For the patients identified as node positive by SLN biopsy, the median number of SLNs identified was 3 (range, 1-8), the median number of metastatic SLNs was 1 (range, 1-2), and the median size of the metastatic focus was $3 \mathrm{~mm}$ (range, $1-5 \mathrm{~mm}$ ). Three of the SLN-positive cases had a prior negative axillary ultrasound examination, yielding an axillary ultrasound false-negative rate of $17 \%$.

Thirty-three $(87 \%)$ of these 38 patients underwent lymphatic mapping in conjunction with the recom-
TABLE 1. Clinicopathologic features of the study patient population $(n=91)$

\begin{tabular}{lc}
\hline Variable & Data \\
\hline Age, y, median (range) & $40(32-75)$ \\
Primary tumor size, cm, median (range) & $3.3(1.5-10)$ \\
Histology & $83(91 \%)$ \\
Invasive ductal & $4(4 \%)$ \\
Invasive lobular & $4(4 \%)$ \\
Mixed invasive ductal and lobular & $53(58 \%)$ \\
Primary pre-neoadjuvant CTX axillary evaluation & $38(42 \%)$ \\
Ultrasound and SLN biopsy results negative & $20(22 \%)$ \\
Axillary node positive & $18(20 \%)$ \\
Ultrasound-guided FNA biopsy & \\
SLN biopsy positive &
\end{tabular}

CTX, chemotherapy; SLN, sentinel lymph node biopsy; FNA, fine-needle aspiration.

mended completion ALND after delivery of the neoadjuvant CTX. Of the remaining five patients, one refused any additional axillary surgery (this patient had undergone pre-neoadjuvant CTX SLN biopsy), and four underwent completion ALND after neoadjuvant CTX was delivered, but without a follow-up SLN biopsy. At 2 years' follow-up, the patient who refused additional axillary surgery had no evidence of locoregional relapse. Of the 4 patients who underwent ALND without follow-up mapping, 3 had no residual nodal metastases, and 1 patient had a single micrometastasis in 1 of 23 nodes.

\section{Completion ALND and Lymphatic Mapping}

Of the 33 patients who underwent completion ALND with follow-up lymphatic mapping, 32 (97\%) of 33 patients underwent a successful SLN biopsy. One patient had a failed mapping, with no SLN identification. This patient had initially been diagnosed as node positive by pre-neoadjuvant CTX SLN and had no residual metastatic disease identified on post-CTX ALND. There was also no uptake of radioisotope (as confirmed by a negative preoperative scan) and no blue dye uptake in this patient. Marked fibrosis was noted during surgery within the axillary tissue, and pathologic assessment revealed an extensive treatment effect. Aside from this nonidentification case, the median number of SLNs identified was 3 (range, $1-10$ ). The median total number of nodes retrieved from the ALND procedure was 10 (range, 6-35).

Of the 32 successfully mapped cases, $22(69 \%)$ had residual axillary nodal disease, and $10(31 \%)$ had a completely negative ALND, with no residual metastases identified. All but 1 of the 22 node-positive cases included a positive SLN node, for a false-negative 
TABLE 2. Final axillary pathology in the subset of neoadjuvant CTX patients found to be node positive at presentation, stratified by the initial method of detecting node metastases

\begin{tabular}{|c|c|c|c|}
\hline \multirow[b]{2}{*}{ Variable } & \multicolumn{3}{|c|}{ Initial method of establishing axillary node metastases } \\
\hline & SLN biopsy & $\begin{array}{l}\text { Ultrasound-guided } \\
\text { FNA biopsy }\end{array}$ & $\begin{array}{c}\text { Either SLN or } \\
\text { FNA (total node-positive cases) }\end{array}$ \\
\hline No. of cases & 18 & 20 & 38 \\
\hline SLN identification rate & $12 / 13(92 \%)^{a}$ & $20 / 20(100 \%)$ & $32 / 33(97 \%)$ \\
\hline No. of cases with no residual axillary metastases after neoadjuvant CTX & $5 / 13(38 \%)$ & $6 / 20(30 \%)$ & $11 / 33(33 \%)$ \\
\hline No. of cases with residual axillary metastases after neoadjuvant CTX & $8 / 13(62 \%)$ & $14 / 20(70 \%)$ & $22 / 33(67 \%)$ \\
\hline No. of cases with residual metastases in SLNs after neoadjuvant CTX & 8 & 13 & 21 \\
\hline No. of falsely negative SLNs after neoadjuvant CTX & $0 / 8(0 \%)$ & $1 / 14(7.1 \%)$ & $1 / 22(4.5 \%)$ \\
\hline
\end{tabular}

CTX, chemotherapy; SLN, sentinel lymph node biopsy; FNA, fine-needle aspiration.

${ }^{a}$ One patient refused additional axillary surgery after delivery of neoadjuvant CTX, and 4 patients underwent completion axillary lymph node dissection (ALND) only, with no lymphatic mapping; in 1 of 13 cases in which lymphatic mapping was attempted, no sentinel lymph node was identified.

rate of $4.5 \%$. This false-negative case was characterized by a single micrometastasis $(<2 \mathrm{~mm})$ within one non-SLN. Of note, there were two cases in which the residual axillary metastatic disease was limited to the SLN, and there were no metastatic non-SLNs.

Eleven $(33 \%)$ of the 33 initially node-positive cases were free of axillary metastases at the time of completion ALND performed after their neoadjuvant CTX. Five of these 11 cases had been diagnosed as node positive at presentation by pre-CTX SLN biopsy, and the remainder had axillary metastases established by ultrasound-guided FNA biopsy.

Results of the post-neoadjuvant CTX lymphatic mapping stratified by the initial (pre-neoadjuvant CTX) means of assessing node positivity are listed in Table 2. The one false-negative case occurred in a patient whose pre-neoadjuvant CTX node-positive status had been determined by ultrasound-guided FNA biopsy. Stratification by method of pre-CTX nodal biopsy (FNA or SLN) revealed two significant findings: (1) a repeat post-neoadjuvant CTX SLN biopsy was feasible in patients who had undergone a pre-neoadjuvant CTX SLN biopsy (as occurred in 12 of 13 cases), and (2) a complete pathologic nodal response to neoadjuvant CTX occurred in $30 \%$ of cases (as documented by the absence of nodal metastases in 6 of the 20 ALND-negative cases that had previously undergone biopsies by ultrasoundguided FNA).

\section{DISCUSSION}

Lymphatic mapping and SLN biopsy has achieved rapid acceptance over the past 10 years in the oncology community as a means of accurately identifying node-negative patients and sparing them the morbidity of a standard ALND. Application of this technology in cases of relatively larger primary breast tumors has been approached more cautiously because of concerns that tumor embolization from a bulky lesion might obstruct and alter lymphatic drainage pathways. This could contribute to identification of an incorrect SLN or to a failed mapping procedure. Findings by Bedrosian et al. ${ }^{16}$ and Chung et al., ${ }^{17}$ however, are quite reassuring. These investigators reported excellent SLN identification rates (99\% and $100 \%$, respectively) and very low false-negative rates (3\% for both studies) in their respective series of 104 patients with tumors at least $3 \mathrm{~cm}$ in size and of 41 patients with $\mathrm{T} 3$ tumors of the breast.

Patients with relatively larger (but resectable) primary breast tumors are increasingly being treated with neoadjuvant CTX, because this sequence improves eligibility for breast preservation via lumpectomy and offers an opportunity to assess sensitivity versus resistance to the selected systemic therapy regimen. Although the optimal scenario would be for appropriately selected patients to reap the benefits of induction CTX as well as minimally invasive axillary surgery, it is essential that the multidisciplinary team adhere to the oncological principles of accurate staging and maximizing locoregional control of disease. Because the effects of neoadjuvant CTX on intramammary lymphatics and axillary metastases are incompletely understood, there has been ongoing debate regarding the question of whether an SLN biopsy should be performed before or after the delivery of induction therapy.

The earliest studies of lymphatic mapping in neoadjuvant CTX patients involved SLN biopsy performed in the posttreatment sequence, at the time of definitive breast surgery. Table 3 tabulates the findings of multiple studies reported to date $e^{10,11-14,18-24}$ in 
TABLE 3. Studies of sentinel lymph node biopsy performed after neoadjuvant chemotherapy

\begin{tabular}{|c|c|c|c|c|c|}
\hline Study & $\mathrm{T}$ status & Sample size & $\begin{array}{c}\text { Sentinel node } \\
\text { identification rate }\end{array}$ & False-negative rate & $\begin{array}{l}\text { Metastases limited to } \\
\text { sentinel node(s) }\end{array}$ \\
\hline Breslin $^{10}(2000)$ & 2,3 & 51 & $85 \%(42 / 51)$ & $12 \%(3 / 25)$ & $40 \%(10 / 25)$ \\
\hline Nason $^{12}(2000)$ & 2,3 & 15 & $87 \%(13 / 15)$ & $33 \%(3 / 9)$ & $\geq 11 \%^{\mathrm{a}}(\geq 1 / 9)$ \\
\hline Haid $^{18}(2001)$ & $1-3$ & 33 & $88 \%(29 / 33)$ & $0 \%(0 / 22)$ & $50 \%(11 / 22)$ \\
\hline Fernandez ${ }^{19}(2001)$ & $1-4$ & 40 & $90 \%(36 / 40)$ & $20 \%(4 / 20)$ & $20 \%(4 / 20)$ \\
\hline \multirow[t]{2}{*}{ Tafra $^{34}(2001)$} & 1,2 & 29 & $93 \%(27 / 29)$ & $0 \%(0 / 15)$ & NR \\
\hline & & T4d (inflammatory) 8 & $75 \%(6 / 8)$ & $40 \%(2 / 5)$ & \\
\hline Stearns $^{20}$ (2002) & 3,4 & Noninflammatory 26 & $88 \%(23 / 26)$ & $6 \%(1 / 16)$ & $24 \%(5 / 21)$ \\
\hline Julian $^{13}$ (2002) & $1-3$ & 34 & $91 \%(31 / 34)$ & $0 \%(0 / 12)$ & $42 \%(5 / 12)$ \\
\hline Miller $^{21}$ (2002) & $1-3$ & 35 & $86 \%(30 / 35)$ & $0 \%(0 / 9)$ & $44 \%(4 / 9)$ \\
\hline Brady $^{15}(2002)$ & $1-3$ & 14 & $93 \%(13 / 14)$ & $0 \%(0 / 10)$ & $60 \%(6 / 10)$ \\
\hline Piato $^{35}$ (2003) & 1,2 & 42 & $98 \%(41 / 42)$ & $17 \%(3 / 18)$ & $0 \%(0 / 18)$ \\
\hline Balch $^{36}(2003)$ & $2-4$ & 32 & $97 \%(31 / 32)$ & $5 \%(1 / 19)$ & $56 \%(10 / 18)$ \\
\hline Schwartz $^{24}(2003)$ & $1-3$ & 21 & $100 \%(21 / 21)$ & $9 \%(1 / 11)$ & $64 \%(7 / 11)$ \\
\hline Reitsamer $^{37}$ (2003) & 2,3 & 30 & $87 \%(26 / 30)$ & $7 \%(1 / 15)$ & $53 \%(8 / 15)$ \\
\hline Mamounas $^{11}(2002)$ & $1-3$ & 428 & $85 \%(363 / 428)$ & $11 \%(15 / 140)$ & $50 \%(70 / 140)$ \\
\hline
\end{tabular}

${ }^{\mathrm{a}}$ Estimated from manuscript.

NR, not reported.

TABLE 4. Results of sentinel lymph node biopsy performed before delivery of neoadjuvant chemotherapy

\begin{tabular}{|c|c|c|c|c|c|}
\hline \multirow[b]{2}{*}{ Study } & \multirow[b]{2}{*}{ Sample size } & \multicolumn{2}{|c|}{ Pre-CTX SLN biopsy results } & \multicolumn{2}{|c|}{ Post-CTX status } \\
\hline & & SLN ID rate & $\mathrm{SLN}^{+}$ & Management strategy & $\begin{array}{l}\text { No. of post-CTX ALNDs } \\
\text { negative for residual metastases }\end{array}$ \\
\hline Zirngibl $^{38}(2002)$ & 15 & $14 / 15(93 \%)$ & $6 / 14(43 \%)$ & $\begin{array}{l}\text { Completion ALND in } \\
\text { SLN }^{+} \text {patients only }\end{array}$ & $6 / 6(100 \%)$ \\
\hline Sabel $^{39}(2003)$ & 24 & $24 / 24(100 \%)$ & $10 / 24(42 \%)$ & $\begin{array}{l}\text { Completion ALND in } \\
\text { SLN }^{+} \text {patients only }\end{array}$ & $3 / 10(30 \%)$ \\
\hline Ollila $^{40}(2003)$ & 22 & $22 / 22(100 \%)$ & $10 / 22(45 \%)$ & $\begin{array}{l}\text { Completion ALND in } \\
\text { all patients }\end{array}$ & $\begin{array}{l}12 \mathrm{SLN}^{-} \text {patients: } 12 / 12(100 \%) \\
10 \mathrm{SLN}^{+} \text {patients: } 6 / 10(60 \%)\end{array}$ \\
\hline
\end{tabular}

CTX, chemotherapy; SLN, sentinel lymph node biopsy; ALND, axillary lymph node dissection.

which the SLN biopsy was performed with a concomitant ALND after neoadjuvant CTX. The first report, by Breslin et al., ${ }^{10}$ from the M.D. Anderson Cancer Center, revealed the presence of a significant learning curve, reflecting the additional technical difficulty of lymphatic mapping related to the CTX effect on soft tissue in the axilla. A parallel learning curve is reflected in Table 3; false-negative rates as high as $33 \%$ were seen in some of the earlier studies with smaller sample sizes, whereas more recent studies reported no false-negative results. The largestscale experience was reported by the National Surgical Adjuvant Breast and Bowel Project B-27 study, in which more than 400 patients underwent lymphatic mapping with concomitant ALND after preoperative doxorubicin and docetaxel treatment. The SLN identification rate was $85 \%$ and the false-negative rate was $11 \%$ in this multicenter study.

Whereas most of the published data regarding SLN biopsy and neoadjuvant CTX have reported on the accuracy of lymphatic mapping performed after the systemic therapy, several breast oncology programs have adopted the practice of axillary staging with an
SLN biopsy before the delivery of induction CTX. Table 4 summarizes the results of the reported studies. Although this sequence subjects the patient to an additional surgical procedure, it does provide more definitive information for stratifying the extent of disease at presentation. The pre-neoadjuvant CTX SLN biopsy provides only limited information regarding the full extent of axillary nodal disease, but it may nonetheless provide useful information to medical oncologists in planning CTX regimens for node-positive versus node-negative disease. Furthermore, radiation oncologists may incorporate this information into their algorithms for defining potential benefit from regional irradiation, because current consensus statements regarding the indications for extended-field and postmastectomy radiotherapy are applicable only to patients undergoing surgery as initial therapy. ${ }^{25}$ The pre-neoadjuvant CTX lymphatic mapping approach is technically feasible (as evidenced by the excellent identification rates); however, accepted practice standards mandate that patients who are SLN positive undergo a completion ALND. In the setting of neoadjuvant CTX, this completion ALND is typically 
performed after the delivery of the induction therapy, concomitantly with the definitive breast operation. A significant proportion of these completion ALNDs will have completely negative results because the preoperative CTX can sterilize axillary metastases in $25 \%$ to $33 \%$ of cases $^{26-28}$ and because metastatic disease is limited to the $\mathrm{SLN}(\mathrm{s})$ in $30 \%$ to $50 \%$ of cases. It can therefore be argued that this approach results in some unnecessary ALNDs.

In our study, 11 (33\%) of the initially node-positive cases were free of axillary disease at the time of ALND (performed after their neoadjuvant CTX). Five of these cases were diagnosed by pre-CTX SLN biopsy. These five patients may have had no additional metastatic non-SLNs, and we therefore cannot definitively comment on whether their negative postCTX ALND is a consequence of chemoresponse. Twenty patients were established as being node positive on the basis of an ultrasound-guided FNA; six $(30 \%)$ of these patients had no residual metastatic nodes in their ALND specimens. This subset does represent the axillary complete response group, and this rate of CTX effect on axillary metastases is consistent with other studies of nodal chemosensitivity.

One of the major benefits of neoadjuvant CTX is the ability to rapidly assess disease chemosensitivity. Patients who experience a complete pathologic response have a statistically superior outcome, ${ }^{29,30}$ thereby confirming that primary tumor response is an excellent surrogate marker for CTX effect on systemic micrometastases. Studies of neoadjuvant CTX have also suggested that pathologic response specifically within axillary metastases is an independent predictor of outcome. ${ }^{26,28,31}$ It is therefore beneficial to be able to distinguish the patients who are node negative at presentation from those whose axillary metastases were sterilized by the induction CTX.

Our study demonstrates that by using a comprehensive and sequential approach to evaluating the axilla, the benefits of pre-neoadjuvant CTX and postneoadjuvant CTX staging can be maximized while subjecting the fewest patients to the morbidity of an ALND. Axillary ultrasonography can be readily incorporated into the baseline imaging of newly diagnosed breast cancer patients and can then reliably guide the radiologist to an appropriate lymph node for FNA biopsy. Malignant cytopathology on FNA biopsy will reliably stage a patient as having metastatic axillary disease, and in these cases an SLN biopsy will be unnecessary. In contrast, published data reveal that axillary ultrasound can be falsely negative in $20 \%$ of cases, ${ }^{32,33}$ and a definitive SLN biopsy is required to avoid understaging the patient's disease. Patients who are definitively proven to be node negative at presentation can then proceed to receive their neoadjuvant CTX for primary breast tumor downstaging, and no additional axillary surgery is necessary.

It might be argued that there is limited benefit to performing the pre-neoadjuvant CTX SLN biopsy in the minority of cases in which the axillary ultrasound is falsely negative, especially because these will frequently be cases of metastatic foci $<5 \mathrm{~mm}$. However, the approximately $20 \%$ false-negative rate of axillary ultrasound is not trivial. For multidisciplinary breast oncology practices in which the team's systemic therapy or adjuvant radiotherapy recommendations are influenced by the nodal status before neoadjuvant CTX, an SLN biopsy will remain necessary to establish node negativity after an axillary ultrasound examination with negative results. Furthermore, many institutions will not have radiologists who are experienced with axillary ultrasonography, and a pre-neoadjuvant CTX SLN biopsy will be the only way to definitively stage the axilla at presentation.

Patients identified as having metastatic axillary disease at presentation (by either FNA or SLN biopsy) proceed to receive their neoadjuvant CTX, but the axilla should be reevaluated pathologically for evidence of residual disease after neoadjuvant CTX. Our findings are consistent with other studies in demonstrating that the induction therapy will sterilize a substantial fraction of nodal metastases. In our study, one third of cases were completely node negative after neoadjuvant CTX. Furthermore, we found that a follow-up SLN biopsy performed in the post-neoadjuvant CTX setting was a feasible and accurate means of identifying the patients with no residual axillary metastases. If our findings are validated by others, then deferral of the completion ALND should be considered; this approach may save an additional $30 \%$ of patients from having to face the morbidity of an ALND.

Future studies should be designed to corroborate our findings. Furthermore, postneoadjuvant axillary ultrasonography should also be considered, because this may represent a minimally invasive means of identifying patients who do harbor residual axillary metastases.

\section{ACKNOWLEDGMENT}

This project was supported in part by an Interdisciplinary Breast Fellowship Grant from The Susan G. Komen Breast Cancer Foundation. 


\section{REFERENCES}

1. Giuliano AE, Kirgan DM, Guenther JM, Morton DL. Lymphatic mapping and sentinel lymphadenectomy for breast cancer. Ann Surg 1994;220:391-8; discussion 398-401.

2. Krag DN, Weaver DL, Alex JC, Fairbank JT. Surgical resection and radiolocalization of the sentinel lymph node in breast cancer using a gamma probe. Surg Oncol 1993;2:335-9; discussion 340 .

3. Albertini JJ, Lyman GH, Cox C, et al. Lymphatic mapping and sentinel node biopsy in the patient with breast cancer. JAMA 1996;276:1818-22.

4. Kim T, Agboola O, Lyman G. Lymphatic mapping and sentinel lymph node sampling in breast cancer (abstract 139). Proc Am Soc Clin Oncol 2002.

5. Reitsamer R, Peintinger F, Prokop E, Rettenbacher L, Menzel C. 200 Sentinel lymph node biopsies without axillary lymph node dissection - no axillary recurrences after a 3-year follow-up. Br J Cancer 2004;90:1551-4.

6. Veronesi U, Paganelli G, Viale G, et al. Sentinel lymph node biopsy and axillary dissection in breast cancer: results in a large series. J Natl Cancer Inst 1999;91:368-73.

7. Bedrosian I, Reynolds C, Mick R, et al. Accuracy of sentinel lymph node biopsy in patients with large primary breast tumors. Cancer 2000;88:2540-5.

8. Haigh PI, Hansen NM, Qi K, Giuliano AE. Biopsy method and excision volume do not affect success rate of subsequent sentinel lymph node dissection in breast cancer. Ann Surg Oncol 2000;7:21-7.

9. Wilke LG, Giuliano A. Sentinel lymph node biopsy in patients with early-stage breast cancer: status of the National Clinical Trials. Surg Clin North Am 2003;83:901-10.

10. Breslin TM, Cohen L, Sahin A, et al. Sentinel lymph node biopsy is accurate after neoadjuvant chemotherapy for breast cancer. J Clin Oncol 2000;18:3480-6.

11. Mamounas E, Brown A, Smith R, et al. Accuracy of sentinel lymph node biopsy after neoadjuvant chemotherapy in breast cancer: updated results from NSABP B-27 (abstract 140). Proc Am Soc Clin Oncol 2002:21.

12. Nason KS, Anderson BO, Byrd DR, et al. Increased false negative sentinel node biopsy rates after preoperative chemotherapy for invasive breast carcinoma. Cancer 2000;89:218794.

13. Julian TB, Dusi D, Wolmark N. Sentinel node biopsy after neoadjuvant chemotherapy for breast cancer. Am J Surg 2002;184:315-7.

14. Tafra L, Verbanac K, Lannin D. Preoperative chemotherapy and sentinel lymphadenectomy for breast cancer. Am J Surg 2001;182:312-5.

15. Brady EW. Sentinel lymph node mapping following neoadjuvant chemotherapy for breast cancer. Breast $J$ 2002;8:97100.

16. Bedrosian I, Reynolds C, Mick R, et al. Accuracy of sentinel lymph node biopsy in patients with large primary breast tumors. Cancer 2000;88:2540-5.

17. Chung M, Ye W, Giuliano A. Role for sentinel lymph node dissection in the management of large ( $>$ or $+5 \mathrm{~cm}$ ) invasive breast cancer. Ann Surg Oncol 2001;8:688-92.

18. Haid A, Tausch C, Lang A, et al. Is sentinel lymph node biopsy reliable and indicated after preoperative chemotherapy in patients with breast carcinoma? Cancer 2001;92:1080-4.

19. Fernandez A, Cortes M, Benito E, et al. Gamma probe sentinel node localization and biopsy in breast cancer patients treated with a neoadjuvant chemotherapy scheme. Nucl Med Commun 2001;22:361-6.

20. Stearns V, Ewing CA, Slack R, Penannen MF, Hayes DF, Tsangaris TN. Sentinel lymphadenectomy after neoadjuvant chemotherapy for breast cancer may reliably represent the axilla except for inflammatory breast cancer. Ann Surg Oncol 2002;9:235-42.

21. Miller AR, Thomason VE, Yeh IT, et al. Analysis of sentinel lymph node mapping with immediate pathologic review in patients receiving preoperative chemotherapy for breast carcinoma. Ann Surg Oncol 2002;9:243-7.

22. Brady E. Sentinel lymph node mapping following neoadjuvant chemotherapy for breast cancer. Breast $J$ 2002;8:97-100.

23. Piato J, Barros A, Pincerato K, Sampaio A, Pinotti J. Sentinel lymph node biopsy in breast cancer after neoadjuvant chemotherapy. A pilot study. Eur J Surg Oncol 2002;29:11820.

24. Schwartz GF, Meltzer AJ. Accuracy of axillary sentinel lymph node biopsy following neoadjuvant (induction) chemotherapy for carcinoma of the breast. Breast $J$ 2003;9:374-9.

25. Recht A, Edge SB, Solin LJ, et al. Postmastectomy radiotherapy: clinical practice guidelines of the American Society of Clinical Oncology. J Clin Oncol 2001;19:1539-69.

26. Kuerer HM, Sahin AA, Hunt KK, et al. Incidence and impact of documented eradication of breast cancer axillary lymph node metastases before surgery in patients treated with neoadjuvant chemotherapy. Ann Surg 1999;230: $72-8$.

27. Newman LA, Buzdar AU, Singletary SE, et al. A prospective trial of preoperative chemotherapy in resectable breast cancer: predictors of breast-conservation therapy feasibility. Ann Surg Oncol 2002;9:228-34.

28. Rouzier R, Extra JM, Klijanienko J, et al. Incidence and prognostic significance of complete axillary downstaging after primary chemotherapy in breast cancer patients with $\mathrm{T} 1$ to $\mathrm{T} 3$ tumors and cytologically proven axillary metastatic lymph nodes. J Clin Oncol 2002;20:1304-10.

29. Kuerer HM, Newman LA, Smith TL, et al. Clinical course of breast cancer patients with complete pathologic primary tumor and axillary lymph node response to doxorubicin-based neoadjuvant chemotherapy. J Clin Oncol 1999;17:460-9.

30. Fisher B, Bryant J, Wolmark N, et al. Effect of preoperative chemotherapy on the outcome of women with operable breast cancer. J Clin Oncol 1998;16:2672-85.

31. Newman LA, Pernick NL, Adsay V, et al. Histopathologic evidence of tumor regression in the axillary lymph nodes of patients treated with preoperative chemotherapy correlates with breast cancer outcome. Ann Surg Oncol 2003;10:734-9.

32. Bedrosian I, Bedi D, Kuerer HM, et al. Impact of clinicopathological factors on sensitivity of axillary ultrasonography in the detection of axillary nodal metastases in patients with breast cancer. Ann Surg Oncol 2003;10:1025-30.

33. Kuenen-Boumeester V, Menke-Pluymers M, de Kanter AY, Obdeijn IM, Urich D, Van Der Kwast TH. Ultrasound-guided fine needle aspiration cytology of axillary lymph nodes in breast cancer patients. A preoperative staging procedure. Eur $J$ Cancer 2003;39:170-4.

34. Tafra L, Verbanac KM, Lannin DR. Preoperative chemotherapy and sentinel lymphadenectomy for breast cancer. $A m J$ Surg 2001;182:312-5.

35. Piato JR, Barros AC, Pincerato KM, Sampaio AP, Pinotti JA. Sentinel lymph node biopsy in breast cancer after neoadjuvant chemotherapy. A pilot study. Eur J Surg Oncol 2003;29:11820.

36. Balch GC, Mithani SK, Richards KR, Beauchamp RD, Kelley MC. Lymphatic mapping and sentinel lymphadenectomy after preoperative therapy for stage II and III breast cancer. Ann Surg Oncol 2003;10:616-21.

37. Reitsamer R, Peintinger F, Rettenbacher L, Prokop E. Sentinel lymph node biopsy in breast cancer patients after neoadjuvant chemotherapy. J Surg Oncol 2003;84:63-7.

38. Zirngibl C, Steinfeld-Birg D, Vogt H, Wagner T, Wischnik A. Sentinel lymph node biopsy before neoadjuvant chemotherapy - conservation of breast and axilla (abstract 516). San 
Antonio Breast Cancer Symposium, San Antonio TX, December 13, 2002.

39. Sabel MS, Schott AF, Kleer CG, et al. Sentinel node biopsy prior to neoadjuvant chemotherapy. Am J Surg 2003;186: $102-5$.
40. Ollila D, Neuman H, Dees E, Sartor C, Metzger R, Carey L. Lymphatic mapping and sentinel lymphadenectomy prior to neoadjuvant chemotherapy in locally advanced breast cancer patients. (abstract 205). 26th Annual San Antonio Breast Cancer Symposium, 2003. 